\title{
TiM: Fine-Grained Rate Adaptation in WLANs
}

\author{
Guanhua Wang, Student Member, IEEE, Shanfeng Zhang, Kaishun Wu, Member, IEEE, \\ Qian Zhang, Fellow, IEEE, Lionel M. Ni, Fellow, IEEE
}

\begin{abstract}
Channel condition varies frequently in wireless networks. To achieve good performance, devices need rate adaptation. In rate adaptation, choosing proper modulation schemes based on channel conditions is vital to the transmission performance. However, due to the natural character of discrete modulation types and continuous varied link conditions, we cannot make a one-to-one mapping from modulation schemes to channel conditions. This matching gap causes either over-select or under-select modulation schemes which limits throughput performance. To fill-in the gap, we propose TiM (Time-line Modulation), a novel 3-Dimensional modulation scheme by adding time dimension into current amplitude-phase domain schemes. With estimation of channel condition, TiM changes base-band data transmission time by artificially interpolating values between original data points without changing amplitude-phase domain modulation type. We implemented TiM on USRP2 and conducted comprehensive simulations. Results show that, compared with rate adaptation choosing from traditional modulation schemes, TiM can improve channel utilization up to $200 \%$.
\end{abstract}

Index Terms-Adapting Interpolation Rate, Modulation Scheme, Rate Adaptation

\section{INTRODUCTION}

W IRELESS communication suffers from continuously varied link condition, which leads to packet loss or bit errors. This time-varying problem is the main issue that limits wireless link's performance. The movement of link nodes and background interference make this time-varying issue even worse. To achieve good channel utilization in such severe conditions, sender needs to select the highest transmission rate that current channel can support, and dynamically adapt the rate to meet with the continuously varied link condition. This procedure is called rate adaptation. Given its widely deployment in WLANs (Wireless Local Area Networks) and mesh networks, rate adaptation plays a vital role in wireless networks.

A large quantity of recent researches advance rate adaptation. Most of them focus on channel condition estimation, like Signal-to-Noise Ratio (SNR) [1] [2] [3], Bit Error Rate (BER) [4] [5]. Based on the channel condition they estimate, they pick up the corresponding modulation scheme for proper transmission rate. Recent literature can estimate channel condition with high accuracy and to some extent, make relatively full use of existed modulation schemes. However, none of them ever focuses on whether existed modulation schemes we choose from are good enough. So here raises a natural question, "can we push the limit of rate adaptation by modifying modulation schemes we choose from and get more throughput gain?"

G. Wang, S. Zhang, Q. Zhang are with the Department of Computer Science and Engineering, Hong Kong University of Science and Technology, Hong Kong, China, (Email:\{gwangab, szhangai, qianzh\}@cse.ust.hk).

L. Ni is with University of Macau. This research was performed when he was with the Department of Computer Science and Engineering, Hong Kong University of Science and Technology, Hong Kong, China, (Email:ni@umac.mo).

$K . W u$ is with the College of Computer Science and Software Engineering, Shenzhen University. He is also with Guangzhou HKUST Fok Ying Tung Research Institute, (Email: kwinson@ust.hk).

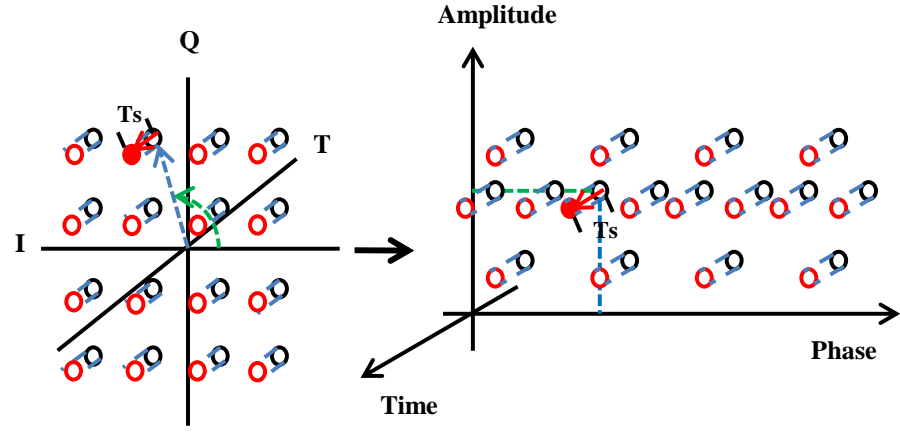

Fig. 1. An example of modified 16-QAM modulation scheme with TiM's additional time dimension.

Properly choosing modulation schemes is a crucial issue in rate adaptation. This choosing process can be regarded as a procedure of mapping different modulation schemes to varied channel conditions. Since the value (e.g. $\mathrm{SNR}$ ) represents channel condition is continuous whereas modulation types are discrete, we cannot make a perfect one-to-one matching from modulation schemes to channel conditions. Because of this non-perfect matching, we may under-select or over-select a scheme that cannot use the bandwidth efficiently. We evaluate the residual between the scheme can utilize and the current channel condition can really support. This link margin is significantly large (details in Section 2.3). Given this modulation gap, we can revise current modulation schemes to move a step forward of rate adaptation and get better channel utilization.

In this paper, we propose a novel modulation scheme Time-line Modulation (TiM), to fix with the non-perfect matching problem. The widely used modulation schemes in WLANs are designed mainly in amplitude-phase domain, such as BPSK (Binary Phase Shift Keying), QPSK (Quadra- 
ture Phase Shift Keying), 16-QAM (Quadrature Amplitude Modulation) and 64-QAM. Based on this 2 Dimension (2D) scheme, TiM adds time dimension to make it into 3D. As depicted in Fig.1, in the traditional 16-QAM modulation scheme, it only has shift in amplitude and phase. The green line refers to the phase shift value whereas the blue line stands for amplitude value. TiM adds time domain as the transmission time of one data symbol, namely Ts, which is shown in Fig.1 as the red arrow. This 2D to 3D expansion can allow us to insert modulation schemes between two existed adjacent schemes (e.g. QPSK and 16-QAM) on time domain without changing anything on traditional amplitude-phase domain.

Some articles also propose to leverage time-domain diversity in modulation [6] [7]. All of them are using time domain diversity to convey information. More precisely, they leverage different time intervals between transmitted symbols to represent information. However, this kind of time-domain leverage indeed cannot improve signal's resistance to noise and interference. In TiM, the length value in time-domain (i.e. Ts) can be adjusted by adapting the quantity of interpolation values that are inserted between real data points. And the interpolation values can enhance emitted signal to be more robust to interference and more likely to be correctly recovered on the receiver side.

TiM can adjust fine-grained level by regulating the quantity of interpolating values between two adjacent sampling data points. However, how to estimate the fine-grained level is a critical issue. Since with higher fine-grained level, rate adaptation process may change modulation schemes more frequently and the changing overhead will be higher. On the other hand, more fine-grained level means more perfect matching of modulation schemes with channel conditions which leads to more throughput gain. Here we design a Grain Size Estimation scheme to make the trade-off between the overhead of changing rate and fine-grained modulation gain.

Since changing modulation schemes on TiM's time domain will have time delay, how to manage this modulation changing overhead is another problem. TiM allows rate changing more efficiently by integrating a simple but useful Lengthen Coordinator module. It leverages processing delay and rate changing delay to cancel each other and reduce the overhead.

One point needs to be claimed is that adding timedimension doesn't mean TiM can achieve real continuous rate adaptation. This is because of two reasons. First, we cannot use a fixed number of bits to represent continuous values which are infinite in system design. Second, there is no need to achieve this which has high overhead without getting significant gain. TiM indeed is also a discrete modulation scheme.

Another point worth mentioning is that while TiM remains the same radio sampling rate, by interpolation, it changes the transmission time of signal symbols, and thereby, causing the signal bandwidth changes accordingly.

We have implemented TiM scheme on USRP2 platform [8]. We also conduct extensive simulations on Matlab for analysing TiM's performance. The results show that, without changing current $2 \mathrm{D}$ modulation schemes, by adding time domain, TiM can achieve up to $200 \%$ goodput com-

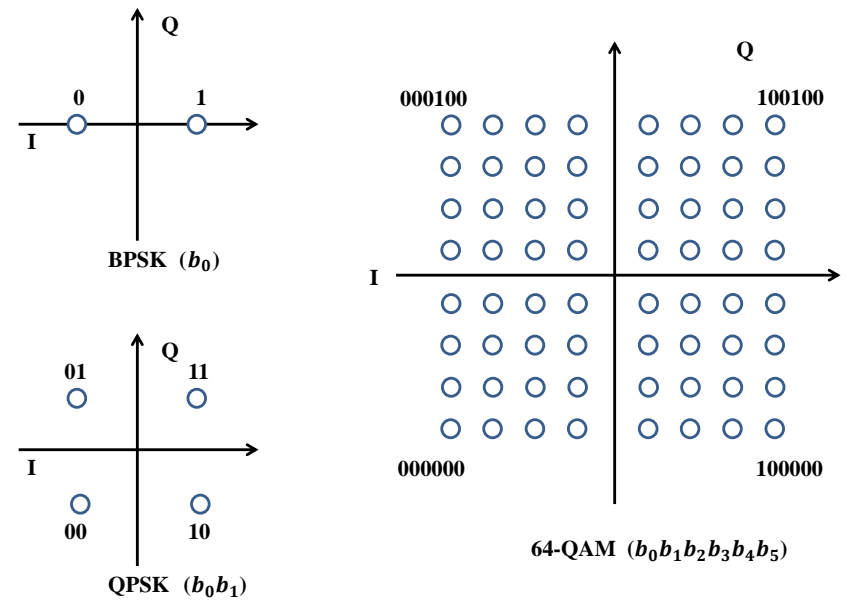

Fig. 2. Basic 2D modulation schemes: BPSK, QPSK and 64-QAM's constellation diagrams.

pared with traditional schemes. For real world wireless traffic, TiM can achieve channel utilization efficiency to nearly $160 \%$ on average. Furthermore, Grain Size Estimation and Lengthen Coordinator's assistance enhances TiM's performance. To sum up, this paper's contributions are mainly as follows.

- To the best of our knowledge, TiM is the first to propose 3D modulation scheme that leverages time domain diversity to fill-in the matching gap of existed $2 \mathrm{D}$ schemes. The primitive is to lengthen the transmission time of base-band data.

- TiM incorporates Lengthen Coordinator to reduce rate changing overhead.

- Grain Size Estimation module is designed for determining TiM's fine-grained level in wireless link to achieve better channel utilization.

- We implemented TiM on USRP2 and modified physical layer (PHY) preamble to make a hand-shake of TiM's interpolation factor between transceivers. Further, we validate and evaluate TiM's performance.

The rest of this paper is organized as follows. In section 2 , we mainly depicts some related preliminaries, motivation of TiM, and a general overview of TiM. Section 3 describes TiM's detailed system architecture. We implement TiM and assistant modules on USRP2 platform in Section 4. We evaluate TiM's performance in Section 5. Section 6 reviews the related work and Section 7 concludes the paper.

\section{Preliminaries and OVerview}

This section depicts some related preliminaries, motivation of TiM, and a general overview of TiM. It first provides some background materials about existed modulation schemes and OFDM primer. Then it delivers the motivation of our TiM approach. Finally it gives a brief introduction of the key ideas in TiM.

In PHY, the sender modulates a sequence of bits into a PHY symbol. There are three basic modulation types which are ASK (Amplitude Shift Keying), PSK (Phase Shift Keying) and FSK (Frequency Shift Keying). In commercial use, namely IEEE $802.11 \mathrm{a} / \mathrm{g} / \mathrm{n}$, what we use in current 
wireless system are BPSK, QPSK, 16-QAM and 64-QAM, etc. These widely-used modulation schemes represent the data bits on a 2D plane which is call constellation diagram.

BPSK and QPSK are both leveraging signals' shifting difference in phase domain. As shown in Fig.2, BPSK uses $180^{\circ}$ phase shift to define data bit 0 and 1 (e.g. when phase shift value $0^{\circ}$ represents bit $0,180^{\circ}$ represents bit 1 or vice versa). It is only able to modulate 1 bit (i.e. $b_{0}$ in BPSK of Fig.2) per symbol. However, it has the highest resistance level to noise or distortion. QPSK refers to that the signal shifts among the phases states are separated by $90^{\circ}$, which is depicted in Fig.2 as QPSK. And each phase shift state conveys two bits data (i.e. $b_{0} b_{1}$ in QPSK of Fig.2), namely $00,01,10,11$, respectively. Since two bits are simultaneously modulated into one symbol, the data transmission bitrate of QPSK is twice as that of BPSK.

16-QAM and 64-QAM use both amplitude and phase shifting to convey more information than either one method alone. The constellation diagram of 16-QAM is shown in Fig.1 without our time-line dimension (i.e. Ts). In each quadrant of this diagrams there are 4 state points with difference in amplitude or phase to distinguish each of them. These 16 state points in the whole diagram convey the bits information from 0000 to 1111 . Thus $16-\mathrm{QAM}$ is a 4 bits per symbol modulation scheme. 64-QAM is quite similar with 16-QAM, it only dense the points in each quadrant from 4 to 16, which can be seen in Fig.2. And each state represents 6 bits per symbol (i.e. $b_{0} b_{1} b_{2} b_{3} b_{4} b_{5}$ in 64-QAM of Fig.2). Since the state points become denser, the signal's resistance to interference or noise is weaker. The data transmitted by this modulation scheme should be in good channel conditions.

\subsection{OFDM Primer}

We present the design of TiM based on OFDM (Orthogonal Frequency Division Multiplexing) which is a widely used technique in WLANs (e.g. IEEE $802.11 \mathrm{a} / \mathrm{g} / \mathrm{n}$ ). OFDM is a multiple subcarriers modulation technique in wireless modulation. It divides the whole bandwidth into several orthogonal subcarriers. Then it spreads data into the lowspeed subcarriers and transmits them in parallel. In current WiFi system, OFDM split a $20 \mathrm{MHz}$ into 64 subcarriers, and each divided subcarrier has $312.5 \mathrm{KHz}$ [9].

In order to modulate base-band data onto OFDM subcarrier, we need the modulation schemes to use constellation symbol to represent data information. Commonly used modulation schemes are BPSK QPSK and so forth. These constellation symbol are transformed into time domain samples through a IFFT process.

To make fully utilization of frequently varied wireless channel condition, Wi-Fi sender employs the technique called rate adaptation. Based on the estimation of channel condition (e.g. SNR), it pick up the densest constellation scheme and highest coding rate to approaching the optimal throughput that current link can support. And recently there are numerous quantity of paper discussing more sophisticated and near-optimal rate adaptation scheme, which may achieve more accuracy and better performance.
TABLE 1

Measurement on SNR threshold for different modulations

\begin{tabular}{|c|c|c|c|}
\hline Min Required SNR & Bitrate & Modulation & Coding \\
\hline $3 \mathrm{~dB}$ & $6 \mathrm{Mbps}$ & BPSK & $1 / 2$ \\
$4 \mathrm{~dB}$ & $9 \mathrm{Mbps}$ & BPSK & $3 / 4$ \\
$5.5 \mathrm{~dB}$ & $12 \mathrm{Mbps}$ & QPSK & $1 / 2$ \\
$9 \mathrm{~dB}$ & $18 \mathrm{Mbps}$ & QPSK & $3 / 4$ \\
$12 \mathrm{~dB}$ & $24 \mathrm{Mbps}$ & 16-QAM & $1 / 2$ \\
$17 \mathrm{~dB}$ & $36 \mathrm{Mbps}$ & 16-QAM & $3 / 4$ \\
$22 \mathrm{~dB}$ & $48 \mathrm{Mbps}$ & 64-QAM & $2 / 3$ \\
$23 \mathrm{~dB}$ & $54 \mathrm{Mbps}$ & 64-QAM & $3 / 4$ \\
\hline
\end{tabular}

\subsection{Motivation: 3D Modulation Scheme With Additional Time Dimension}

For many years, in information theory, there are basically three digital modulation types, namely PSK, ASK and FSK. PSK leverages phase shifting value to convey data, whereas ASK uses amplitude difference and FSK uses frequency shifting value. However, in IEEE 802.11 standards [9], basically, the modulation schemes (e.g. BPSK, 16-QAM) are all designed in the phase-amplitude two dimensions.

The reason why we do not leverage FSK or frequency domain schems is because the usage of OFDM. OFDM is widely deployed in current WiFi communication system owing to its high efficiency of channel utilization. However, OFDM is not perfect. The shortage is that OFDM is strict with subcarries' orthogonality and time synchronizing. If we implement FSK into OFDM, it will decrease the synchronizing performance in OFDM. Further, Adding frequency domain modulation types also needs the offset estimation of doppler effect to be more precise. Because of these two main reasons, we cannot use FSK in current modulation schemes.

Even though each 2D modulation scheme (e.g. BPSK) has few coding rate (e.g. 1/2 3/4) options, it cannot achieve decent performance [10]. There are mainly two reasons. First, due to the limited number of pre-defined options, traditional 2D scheme cannot achieve varied fine-grained level modulation whereas TiM can. Second, TiM indeed can be implemented with any coding rate and make each of these options be more fine-grained which enhance the performance.

Because the non-perfect matching between existed modulation schemes and channel conditions, it causes throughput loss when changing modulation schemes. Nevertheless, conventional 2D modulation schemes have been studied for many years and have already been perfectly designed. So how can we fill-in the matching gap without modifying the well-defined amplitude-phase domain schemes? Additionally, we cannot directly use the frequency domain schemes either.

Given all these concerns and analysis above, the only way we can modulate the signal besides amplitude-phase 2D domain is to design modulation schemes that leverage the time domain. This expansion from $2 \mathrm{D}$ to $3 \mathrm{D}$ is a way that we can fill-in the matching gap between current 2D modulation schemes and channel conditions. 


\subsection{TiM Modulation Framework}

The goal of TiM is to cover the matching gap between adjacent modulation schemes with negligible overhead.

As shown in table 1, we measured the corresponding SNR threshold on USRP2 [8] platform for different modulation schemes with varied coding rates. The SNR threshold measurement is based on packet loss rate. More precisely, when the packet loss rate is above a threshold (empirically $30 \%$ ) in a specific transmission bitrate, the sender regards current SNR cannot support this particular transmission rate. This measurement is based on fact that rate adaptation algorithm tries its best to minimize the packet loss rate, thus can be conservative [7].

In addition, we do not use the well-designed rate adaptation schemes. It is because that using more sophisticated schemes to measure the channel SNR will increase the measurement overhead. However, the measurement in [7] simulates the state-of-the-art rate adaptation scheme, namely SoftRate [11]. Their observation results also support our link margin statements.

Based on our experimental results on USRP2 in table 1 , for example, when the estimated channel SNR is in the interval of minimum SNR requirement of two adjacent modulation schemes (e.g. $15 \mathrm{~dB}$ ), choosing either of these two schemes (i.e. 16-QAM with $1 / 2$ coding rate or 16-QAM with $3 / 4$ coding rate) cannot fully utilize the channel. And the residual between selected scheme can utilize and the throughput that channel can really support is a significant margin that we want to utilize. By filling in this matching gap, we can reduce throughput loss of rate changing process. To this end, we need to ensure that the newly designed modulation scheme can be inserted exactly between the two traditional adjacent schemes it based on.

TiM's insight is to utilize the modulation's time domain without disturbing current 2D (amplitude and phase domain) scheme. To do so, trigged by [12] [13] which slowdown clock rate to reduce power consumption, we lengthen the data symbol transmission time by adding some interpolating points between adjacent original data symbols. This interpolation process is easy to be added in current OFDM modulation procedure and can be easily implemented on any coding schemes. We realize this function by modifying the CIC (Cascaded Integrator-Comb) circuits in transceivers.

\section{TIM DESIGN}

In this section, we describe the detailed design of TiM. Before theoretical analysis of why TiM works, we first present the overview of system architecture. As depicted in Fig.3, TiM's transceivers mainly consist of 3 parts, namely Grain Size Estimation, TiM modulation/demodulation and Lengthen Coordinator. The main working procedure is as follows. First, with channel variation information, by using Grain Size Estimation, we set proper fine-grain level to balance between the overhead of changing rate and fine-grained modulation gain. Then, we implement TiM modulation/demodulation for upper layer data encoding/decoding and leverage Lengthen Coordinator to reduce the rate changing head.

In the following parts of this section, we mainly focus on making a theoretical proof of why TiM can improve Packet

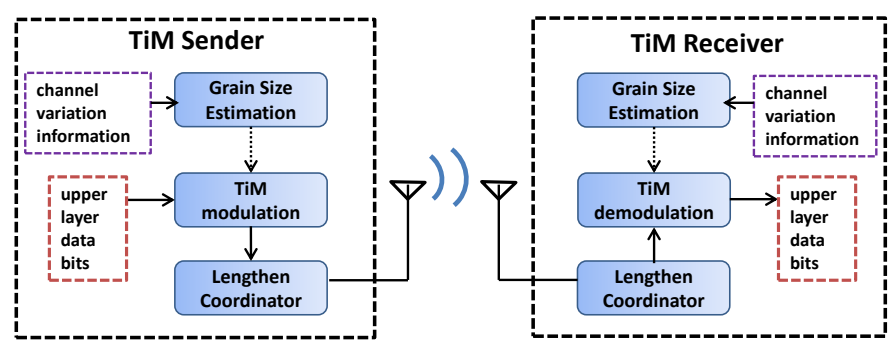

Fig. 3. High-level architecture of TiM's sender and receiver.

Reception Rate (PRR) or throughput without changing the basic 2D scheme. Further, we discuss how well TiM can increase signal symbol's robustness by quantifying SNR gain that TiM can achieve in Additive White Gaussian Noise (AWGN) channel. Then we deliver the concrete design of TiM's modulation scheme and demodulation scheme separately.

\subsection{Design Principles of TiM}

In this part, we prove that TiM can improve PRR by strengthening signal's robust to noise and interference. We formally derive TiM's performance by modeling how the signal transmitted through the channel and then recovered at the receiver. We list some basic concepts and notations that we may use during the theoretical analysis and following sessions in table 2 .

Let $u[k]$ refers to the convolution value of the symbol emitted from sender $x_{s}[k]$ and the corresponding channel parameter $h[k]$. During the $k^{t h}$ symbol transmission, the noise parameter is $n[k]$. The received symbol signal $y_{r}[k]$ is as equation 1 ,

$$
y_{r}[k]=u[k]+n[k]=x_{s}[k] * h[k]+n[k]
$$

Due to the limited pages, here we do not depict a figure of the receiver's CIC circuit, which is similar to the CIC circuit on the sender side in Fig.6. The difference between sender's and receiver's CIC circuit is just the a place exchange of integrator module and comb module (i.e. For the receiver's CIC circuit, on R module's left-hand side is integrator module, whereas on $\mathrm{R}$ module's right-hand side is comb module). Additionally, the $\mathrm{R}$ module in the receiver is used for decimation which is just the inverse operation of $\mathrm{R}$ module on the sender side. The received signal first pass through integrator module, then pass decimator (i.e. R module) and finally pass the comb module of CIC. Normally, in CIC circuit, we have integrator and comb with 4-stage as shown in Fig.6.

Definition $1\left(x_{i N}(k)\right)$. On the CIC circuit of the receiver side, let $x_{i N}$ denotes the integrator module's output of the $k^{t h}$ symbol passing through this circuit. The letter $N$ refers to the number of stages in this integrator module, whereas letter $i$ stands for integrator.

Definition $2\left(x_{d}(k)\right)$. Given the output of integrator $x_{i N}$, the data symbol need to pass through the decimator (i.e. $\mathrm{R}$ module). $x_{d}(k)$ denotes the decimator's output of $k^{\text {th }}$ symbol, where $d$ represents the meaning of decimator. 
TABLE 2

Concepts and notations

\begin{tabular}{|c|c|}
\hline$N$ & Number of subcarries \\
\hline$R$ & Interpolation/Decimation factor of CIC circuit \\
\hline$h[k]$ & Channel parameters of the $k^{t h}$ symbol \\
\hline$n[k]$ & Noise parameter of the $k^{t h}$ symbol \\
\hline$x_{s}[k]$ & the $k^{t h}$ signal symbol emitted from sender \\
\hline$y_{r}[k]$ & the $k^{t h}$ signal symbol received at receiver side \\
\hline$\omega_{k}$ & wight value for each data sample after pass CIC circuit \\
\hline$P$ & Signal transmission power \\
\hline$\xi_{s n r}$ & SNR gain after we implementing modified CIC circuit \\
\hline
\end{tabular}

Definition $3\left(y_{c N}(k)\right)$. Given $x_{i N}(k)$ 's definition, here $y_{c N}(k)$ is quiet the same. Let $y_{c N}$ denotes the signal output of comb module. The letter $N$ share the same meaning as in $x_{i N}(k)$, whereas letter $c$ stands for comb. The letter $k$ represents the meaning that it is the $k^{t h}$ symbol passing through this circuit.

Given these definitions above, we first illustrate a simple model, which the receivers CIC filter only contain 1 stage of integrator, then decimator and 1 stage of decimator. As we have mentioned before, $u[k]$ denotes the emitted symbol that received at receiver side with a distortion of $h[k]$. So after passing the 1 stage integrator, we can get the output of integrator as equation 2 . In equation 2 , we can see that the $k^{t h}$ symbol also conveys the $(k-1)^{t h}$ symbol's information, thus make it robust for the receiver to recover the data symbols.

$$
x_{i 1}(k)=x_{i 1}(k-1)+u(n)=\sum_{k=0}^{n} u(k)
$$

After this 1 stage integrating, the symbol need to pass through the decimator (i.e. $\mathrm{R}$ module). Suppose the decimating factor is $R$, the output of decimator is as equation 3.

$$
x_{d}(m)=x_{i 1}(m R)=\sum_{k=0}^{m R} u(k)
$$

After that, the signal should pass through 1 stage comb, the output of comb circuit is as equation 4 . Thus, after this process, it can get the original data symbols' information (i.e. $u(k))$ by doing a subtraction between two adjacent decimated symbols.

$$
\begin{aligned}
y_{c 1}(m) & =x_{d}(m)-x_{d}(m-1) \\
& =\sum_{k=0}^{m R} u(k)-\sum_{k=0}^{(m-1) R} u(k) \\
& =\sum_{k=(m-1) R+1}^{m R} u(k)
\end{aligned}
$$

The theoretical analysis of 1-stage CIC circuit in time domain is delivered as above. There are mainly 3 components in the real 4-stage receiver's CIC circuit. Based on this simple model mentioned above, we show the real 4stage CIC circuit's output by deliver the results of all its three parts separately. The output of the $4^{\text {th }}$ integrator is as equation 5,

$$
x_{i 4}(n)=\sum_{k_{1}=0}^{n} \sum_{k_{2}=0}^{k_{1}} \sum_{k_{3}=0}^{k_{2}} \sum_{k_{4}=0}^{k_{3}}\left[u\left(k_{4}\right)\right]
$$

we can get the output of decimator module as equation

$x_{d}(m)=\sum_{k_{1}=0}^{m R} \sum_{k_{2}=0}^{k_{1}} \sum_{k_{3}=0}^{k_{2}} \sum_{k_{4}=0}^{k_{3}}\left[u\left(k_{4}\right)\right]$ where $m \in\left[0,\left\lfloor\frac{n}{R}\right\rfloor\right]$

After that, the signal should pass through 4-stage comb module. We can derive the final output of this whole 4-stage CIC circuit as equation 7 ,

$$
y_{c 4}(m)=\sum_{k_{1}=(m-4) R+4}^{(m-3) R+3} \sum_{k_{2}=k_{1}}^{k_{1}+R-1} \sum_{k_{3}=k_{2}}^{k_{2}+R-1} \sum_{k_{4}=k_{3}}^{k_{3}+R-1} u\left(k_{4}\right)
$$

Given the noise parameter as $n(k)$, the output of noise $y_{n}$ passing through CIC can be derived from the output of signal passing through $\mathrm{CIC}$ as equation 8 .

$$
y_{n}(m)=\sum_{k_{1}=(m-4) R+4}^{(m-3) R+3} \sum_{k_{2}=k_{1}}^{k_{1}+R-1} \sum_{k_{3}=k_{2}}^{k_{2}+R-1} \sum_{k_{4}=k_{3}}^{k_{3}+R-1} n\left(k_{4}\right)
$$

Based on the output of CIC circuit (i.e. equation 7), we can get that the CIC output is the weighted (i.e. $\omega_{k}$ ) sum of the last $4(R-1)$ input samples, which can be delivered as the equation 9 ,

$$
y_{s}(m)=\sum_{k=(m-4) R+4}^{m R} \omega_{k} u(k)
$$

Let's first calculate the SNR of wireless system without the CIC filter procedure. Suppose noise is modeled as AWGN, $n(k)$ meets with the Gaussian distribution. Thus we can derive that,

$$
n(k) \sim N\left(0, \delta^{2}\right)
$$

The SNR (i.e. $\xi_{s n r}^{\prime}$ ) can be represented as equation 10, where $P$ is the signal transmission power,

$$
\xi_{s n r}^{\prime}=\frac{E(u(k)-E(u(k)))^{2}}{E(n(k)-E(n(k)))^{2}}=\frac{P}{\delta^{2}}
$$

When implementing CIC circuit with the interpolating factor of $R$ at the sender side, the SNR gain (i.e. $\xi_{\text {snr }}$ ) at the receiver can be depicted as equation 11. And we can easily derived that the SNR gain is larger than $R \frac{P}{\delta^{2}}$. Additionally, the simulation result from Matlab in Fig. 4 also verifies and supports our theoretical analysis.

Furthermore, in Fig.4, with the higher value of interpolation factor, we get more unstable SNR gain (as the error bar's length is increasing). The reason for this phenomenon is that, since noise obeys Gaussian distribution, the more points we collect, the more Gaussian distribution-like that the noise will be. However, noise that combined with interpolation values and real data symbols is independent, 


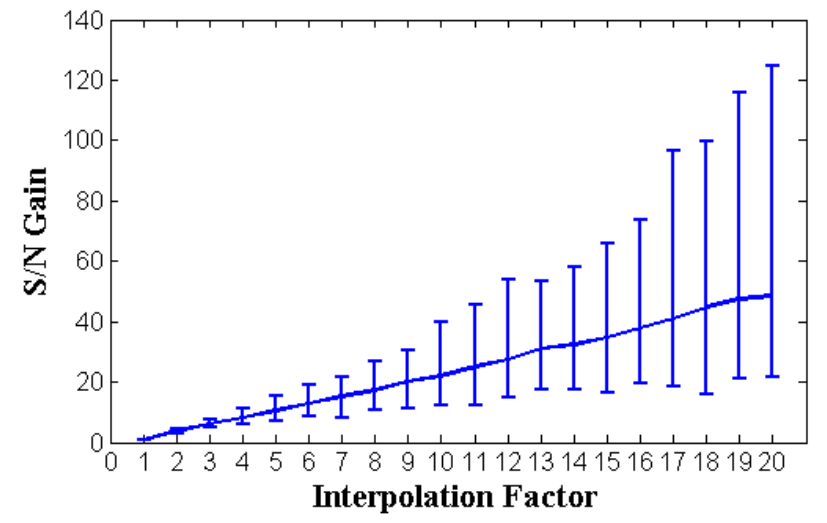

Fig. 4. Relationship of SNR gain and Interpolation/Decimation factor in AWGN channel.

whereas the interpolation values and real data symbols are correlated. After we leverage the interpolation points to help recover the real data points on the receiver side, we throw them away. Due to noise's independent attribute, noise information on the interpolation points indeed is lost. Thus it could be less likely of Gaussian distribution. And this is the reason for unstable SNR gain we get at high interpolation/decimation rate.

$$
\begin{aligned}
\xi_{s n r} & =\frac{E\left(y_{s}^{2}(m)\right)}{E\left(y_{n}^{2}(m)\right)} \\
& \geq \frac{R \sum_{k=(m-4) R+4}^{m R} \omega_{k}^{2} E\left(u^{2}(k)\right)}{\delta^{2} \sum_{k=(m-4) R+4}^{m R} \omega_{k}^{2}}=R \frac{P}{\delta^{2}}
\end{aligned}
$$

Based on the proof above, we can derive that, TiM's interpolating system can help the data signal to be more robust to noise and interference. The more interpolated values between adjacent data points, the better recovery of data points at the receiver side.

\subsection{D Modulation/Demodulation Schemes}

To realize TiM, there are several challenges. First, at the sender side, the lengthen process of base band data must overcome the following difficulties: (1) it must be configurable to set different lengthen value during the rate adaptation process. (2) how to determine the lengthen value with varied channel condition is an open problem. We deliver our methods to deal with these two problems from both theoretical analysis and Matlab simulation results.

On the receiver side, the design of demodulation scheme can be more challenging. The first issue is the delay of changing interpolation/decimation rate between sender and receiver. We present Lengthen Coordinator to solve this problem. Another difficulty is how to recover data points with less accurate coordination and synchronization between the sender and the receiver. To handle this problem, we leverage the interpolated points to help improve the accuracy of data recovery.

\subsubsection{Modulation Scheme Design}

The key idea of the Time-line modulation scheme is to lengthen the transmission time of base-band data. To do

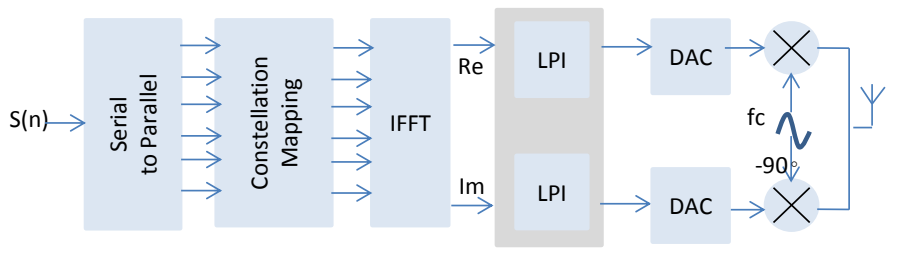

Fig. 5. Sender side OFDM system structure with Low Pass Interpolation (LPI) block. TiM modified its CIC (Cascaded Integrator-Comb) filter to adjust interpolation parameter.

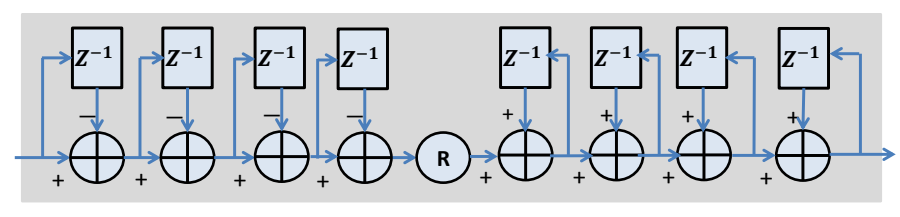

Fig. 6. Inner structure of TiM's Modified CIC circuit in sender's LPI block.

so, we need to interpolate redundancy values between any two adjacent original data points we need to transmit. As illustrated in Fig.5, we modified the CIC circuits on sender side's low pass interpolator (LPI) [14] in order to change interpolation factor dynamically. With this modified LPI, based on the estimation of current SNR, we can decide how many interpolation values we need to insert between adjacent real data points.

First we make an analysis about this modification in order to illustrate this process more clearly. Let the data symbols we want to transmit be the $\{X[k]\}$ set. For each data symbol $x[n]$, the output of IFFT (Inverse Fast Fourier Transform) is as equation 12 ,

$$
x[n]=\frac{1}{N} \sum_{k=0}^{N-1} X[k] e^{j 2 \pi \frac{k n}{N}}
$$

In Fig.5, after the IFFT process at the sender side, the plural data symbol $x[n]$ we need to transmit is separated into two flows, namely real part $(\operatorname{Re})$ and imaginary part (Im). After that, each of these two symbol flows (i.e. Re $x[n]$ and $\operatorname{Im} x[n])$ are independently passing through the LPI module with an interpolation factor. Here we use $R$ to denote the interpolation factor.

As shown in Fig.6, the CIC filter here is specially designed. It contains a 4-stage cascaded integrators, an interpolation module and a 4-stage comb. We modified the CIC filter in order to make the interpolating factor can be reprogrammed. The main duty of this module is used for interpolation by using Z-domain transformation. The lefthand side which is before module "R" in Fig.6, it is called comb module. Let $y_{1}[z]$ and $x_{1}[z]$ denote the output and input of this part circuit, respectively. The Z-domain transfer function $H_{C}(z)$ is illustrated as equation 13 ,

$$
H_{C}(z)=\frac{y_{1}(z)}{x_{1}(z)}=\left(1-z^{-1}\right)^{4}
$$

Integrator module is on the right-hand side of module "R". $y_{2}[z]$ and $x_{2}[z]$ also denote the output and input of 


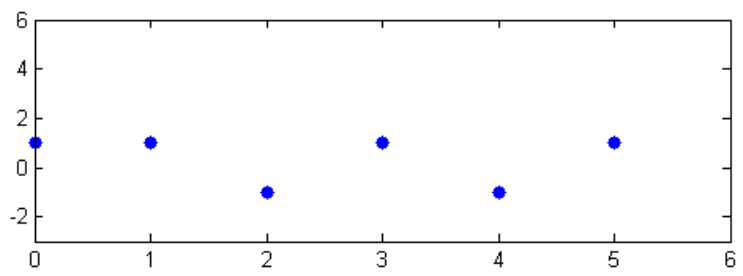

(a) Original data points.

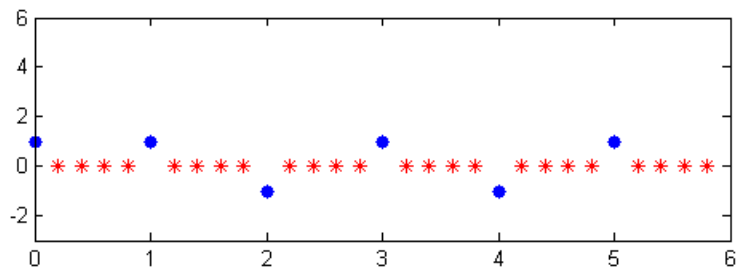

(b) Original data points with artificial interpolated points.

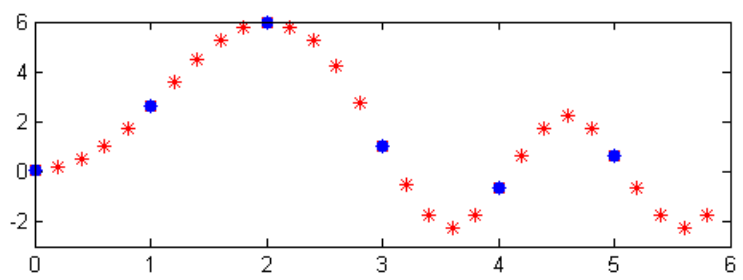

(c) Output of data points and interpolated points pass through LPI circuit

Fig. 7. The process of interpolating points between adjacent data points pass through LPI circuit, where blue points stand for original data points and red stars denote interpolation points.

this part circuit, respectively. The corresponding Z-domain transfer function $H_{I}(z)$ is as equation 14,

$$
H_{I}(z)=\frac{y_{2}(z)}{x_{2}(z)}=\left(\frac{1}{1-z^{-1}}\right)^{4}
$$

The function of the modified " $\mathrm{R}$ " module is to do interpolation process, which can be represented as equation 15 . Here $y_{3}[z]$ and $x_{3}[z]$ also denote the output and input of this part circuit.

$$
y_{3}[n]= \begin{cases}x_{3}\left[\frac{n}{R}\right] & \text { if } n=k R \\ 0 & \text { other }\end{cases}
$$

One point needs to be explained. The value we artificially interpolated between the data sampling points has the initial value of 0 . Because of the special characteristic of the CIC filter, the interpolated 0-value points can be changed into redundancy values which are helpful for recovering data at the receiver side. For more details, we suggest to read [15] and [16] which have detailed theoretical explanation of CIC filter technique.

Based on these basic equations, We can derive the whole circuit's Z-domain transfer function as equation 16,

$$
\begin{aligned}
H(z) & =H_{c}\left(z^{R}\right) \times H_{I}(z) \\
& =\left(1-z^{-R}\right)^{4} \times\left(\frac{1}{1-z^{-1}}\right)^{4} \\
& =\left(\frac{1-z^{-R}}{1-z^{-1}}\right)^{4}
\end{aligned}
$$

According to this Z-transfer function in equation 16, we transfer it into time-domain and get the input $x[n]$ and output $y[n]$ of this whole modified LPI as equation 17 ,

$$
\begin{aligned}
y[n] & =4 y[n-1]-6 y[n-2]+4 y[n-3]-y[n-4] \\
& +x[n]-4 x[n-R]+6 x[n-2 R]-4 x[n-3 R] \\
& +x[n-4 R]
\end{aligned}
$$

Based on equation 17, we can change the length of each data symbol's transmission time by interpolating values between adjacent data points. Furthermore, the artificially added redundancy points will help the signal to be more robust to interference and noise during transmission and can be better recovered at the receiver, which has been proved in section 3.1. To make it clear, the whole process of interpolating 0 -value points and finally get the redundancy values is represented as Fig.7. As depicted in Fig.7, the numbers on the horizontal axis refer to the sequential numbers of each original data point. The numbers on the vertical axis denote the corresponding values of each interpolating and original data point.

Another challenge we need to conquer is how to set the specific lengthen value to deal with varied channel condition. Based on Shannon's theorem, we first make a theoretical analysis about the relationship between the lengthen value and signal's resistance to noise and interference.

From the information theory, the channel capacity (i.e. C) can be represent as equation 18 ,

$$
C=B \log _{2}\left(1+\frac{S}{N}\right)
$$

Additionally, we can derive that, for a given SNR with one fixed modulation scheme, we have the relationship between highest throughput (i.e. T) and channel capacity as depicted in equation 19 ,

$$
T \propto C
$$

Based on this, since the interpolation factor $R$ is inversely proportional to the throughput $T$, we can derive the relation between interpolation factor $R$ and $\frac{S}{N}$ as equation 20, where $T_{n e w}$ denotes the throughput of changed interpolation scheme with new interpolation value $R_{\text {new }}$. And $T_{\text {ori }}$ stands for the original throughput with the initial interpolation value $R_{\text {ori }}$. $S_{\text {new }}$ is the signal strength of changed interpolation factor whereas $S_{\text {ori }}$ is signal strength with the original interpolation factor.

$$
\frac{R_{\text {ori }}}{R_{\text {new }}}=\frac{T_{\text {new }}}{T_{\text {ori }}}=\frac{C_{\text {new }}}{C_{\text {ori }}}=\frac{B \log _{2}\left(1+\frac{S_{\text {new }}}{N}\right)}{B \log _{2}\left(1+\frac{S_{\text {ori }}}{N}\right)}
$$

Now we can draw the conclusion that, given a fixed bandwidth, $R$ is inversely proportional to $\log _{2}\left(1+\frac{S}{N}\right)$. In other words, given one fixed bandwidth, the more interpolation points being inserted, the lower SNR needed for the receiver to correctly recover the real data symbols.

Further, our Matlab simulation result in Fig.8 tests and verifies our theoretical analysis. We simulated TiM in AWGN channel. And we built up the mapping rules of interpolation/decimation factor and its corresponding SNR, for commonly used modulation schemes like BPSK QPSK, 16-QAM, 64-QAM. Based on this relationship, we can map 


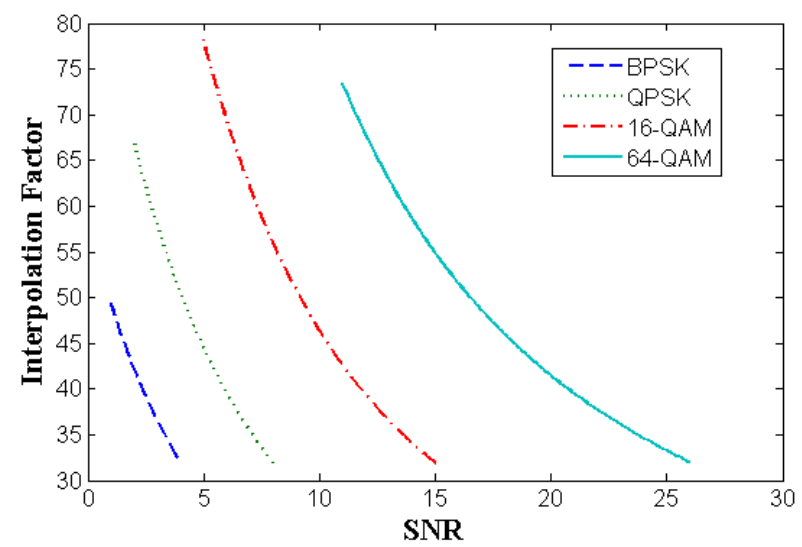

Fig. 8. Mapping rules for interpolation/decimation factor with varied SNR.

the specific lengthen value (i.e. interpolation factor) to the varied channel conditions.

\subsubsection{Demodulation Scheme Design}

In TiM's demodulation process, it is not just a reverse procedure of the modulation process. The design of TiM's demodulation scheme seems to be more challenging. And there are mainly two following difficulties.

First, when changing the interpolation rate, the coordination between transceivers is a hard problem. How to manage this procedure in order to minimize the overhead of rate changing delay? The module "Lengthen Coordinator" we proposed in section 4 can solve this problem with negligible overhead.

Second, when SNR varies frequently, our interpolation rate changing may also be frequent. Given this, the receiver's sampling rate may not be perfectly synchronized and coordinated with the sender's. There may exists difference between the original data points and receiver's sampled values. Own to our interpolation points, this distortion can be recovered by TiM's interpolated value. This recovery procedure can be regarded as another aspect of enhancing signal's robust attribute that TiM can achieve.

\section{IMPLEMENTATION}

In this section, we present a detailed implementation of TiM. We use GNU radio [17] to implement TiM's sender and receiver on USRP2 software radio platform [8]. As depicted in Fig.3, TiM consist of 3 parts, namely Grain Size Estimation, TiM modulation/demodulation and Lengthen Coordinator. As mentioned in previous section III, the main working procedure can be illustrated as follows. First, with channel variation information, by using Grain Size Estimation, we set proper fine-grain level to balance between the overhead of changing rate and fine-grained modulation gain. Then, we implement TiM modulation/demodulation for upper layer data encoding/decoding and leverage Lengthen Coordinator to reduce the rate changing head.

In following parts, We first deliver the implementation procedure of the TiM's modulation/demodulation design on the sender and the receiver. After that we discuss about Lengthen Coordinator and Grain Size Estimation design.

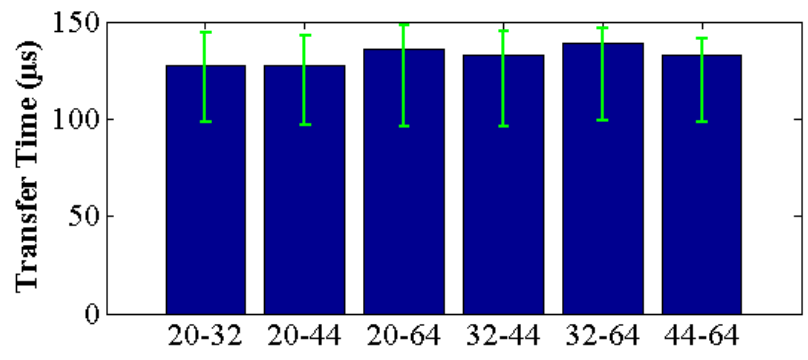

(a) Time delay of increasing interpolation.

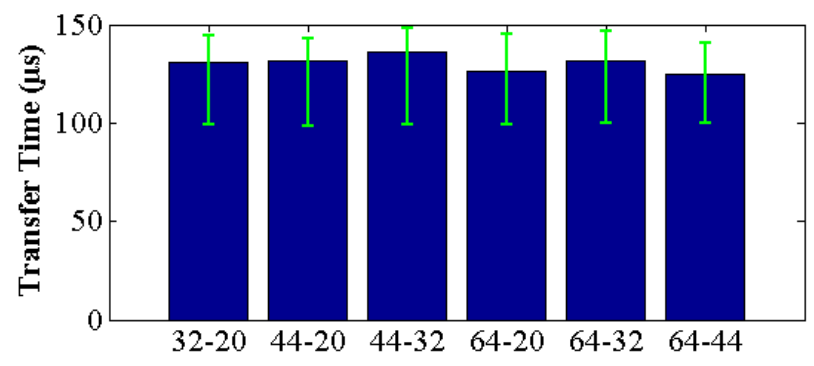

(b) Time delay of decreasing interpolation.

Fig. 9. Time delay in changing interpolation rate.

\subsection{TiM's Modulation/Demodulation Scheme Imple- mentation}

As described in section 3.2, we need to modify module " $R$ " in the CIC circuit of transceivers to enable that the interpolation/decimation factor can be reprogrammed. After this modification, we artificially insert the interpolation points (with initial value of 0 ) before the interpolation process. Then we can change the interpolation factor based on the number of interpolation points we insert between two adjacent real data points. We let the data points and inserted interpolation points together pass through the CIC circuit and finally get the combined modulated signal as the CIC's output. Then we transmits the modulated signal to the receiver.

On the receiver side, we demodulate the combined signal as an inverse process of the modulation procedure on the sender side. After we decoded one specific data point and its correlated interpolation values, we leverage the interpolation values to make an estimation of this data point to enhance the accuracy of data recovery. Since there are huge amounts of value estimation algorithms (such as belief propagation in [18], confidence level in [19] and so on), here we use the K-means clustering algorithm [20] to do this estimation.

\subsection{PHY Preamble Modification}

In order to coordinate the transceiver's interpolation/decimation rate, we need to modify PHY preamble. In IEEE 802.11 standard [9], modulation scheme information is contained in the RATE field of OFDM PLCP (Physical Layer Convergence Protocol) preamble.

The RATE field of OFDM PLCP preamble consists of 4 bits, which can represent at most 16 modulation schemes. The current modulation schemes in use are BPSK QPSK 16QAM with coding rate of $1 / 2$ and $3 / 4$, and 64-QAM with coding rate of $2 / 3$ and $3 / 4$. Therefore, there are $16-8=8$ 
empty positions can be used for representing additional modulation schemes provided by TiM. As for normal situations, we believe that adding 8 modulation schemes is fine-grained enough to get the near optimal goodput in a wireless link. So here we leverage these 8 available positions in RATE field to represents interpolation factor of 8 additional TiM's modulation schemes. Furthermore, given more fine-grained scenarios, we can modify PHY preamble by adding additional bits on RATE field to represent a larger number of more fine-grained modulation schemes in TiM.

\subsection{Lengthen Coordinator}

We now present the design of Lengthen Coordinator module, which is used for reducing the overhead of rate changing. Basically, there are two main changing overheads. One is the delay in the rate (interpolation factor) changing process. Another is the delay of processing received data at the receiver side. We first discuss these two delays respectively. Then we present Lengthen Coordinator's scheduling algorithm to solve these two issues.

\subsubsection{Delay in Changing Interpolation Rate}

When changing the transmission time of each data symbol, the radio need to be stabilized before transmitting or receiving signals. According to our experimental results in Fig.9(a) and Fig.9(b), the average changing delay is nearly 130us. More precisely, the delay of changing rate varies from 118us to 140us. In addition, Fig.9(a) and Fig.9(b) also depict that changing rate between two specific interpolation factors has different delay. For example, the delay of increasing interpolation factor from 44 to 64 is longer than the delay of decreasing from 64 to 44 . Given this delay issue, We need to manage this delay time, so that it has less probability of disturbing the current wireless transmission during the changing rate process.

\subsubsection{Delay of Received Signal Processing}

On the other side, there is another interesting problem. When we let the sender change the interpolation rate after sending the 1000th packet, however, every time the receiver can only decode the first 994 packets. So why the last six packets cannot be decoded correctly?

To answer this question, we change the interpolation rate changing time of the sender. First, we set the rate changing time after the sender transmits the 500th packet, then after the 1000th, and then after the 2000th packet, so on and forth. The quantity of packets that cannot be decoded is the same. We measure the time point of sender sending packets and receiver receiving packets. The data in table 3 shows the time of emitting and receiving packets where sender changes rate on the 501th packet.

In table 3, there is a time gap between the sender sending one packet and the receiver receiving that specific packet. This is because processing delay on both sender and receiver (e.g. packet 494, the delay between sender and receiver is $28.43-28.35=0.08 \mathrm{~ms}$ ). And this is the key reason why receiver cannot decode the last 6 packets with unchanged interpolation rate.

As in table 3, when the receiver finish processing the 494th packet, it is the same time that the sender sends the
TABLE 3

Packet transmission delay between transmitter and receiver

\begin{tabular}{|c|c|c|}
\hline Packet number & $\begin{array}{c}\text { Sender transmitted } \\
\text { time }(\mathrm{ms})\end{array}$ & $\begin{array}{c}\text { Receiver received } \\
\text { time }(\mathrm{ms})\end{array}$ \\
\hline 491 & 26.52 & 26.6 \\
\hline 492 & 27.11 & 27.2 \\
\hline 493 & 27.73 & 27.81 \\
\hline 494 & 28.35 & 28.43 \\
\hline 495 & 29.09 & $\mathrm{~N} / \mathrm{A}$ \\
\hline 496 & 29.64 & $\mathrm{~N} / \mathrm{A}$ \\
\hline 497 & 30.18 & $\mathrm{~N} / \mathrm{A}$ \\
\hline 498 & 30.76 & $\mathrm{~N} / \mathrm{A}$ \\
\hline 499 & 31.37 & $\mathrm{~N} / \mathrm{A}$ \\
\hline 500 & 32.03 & $\mathrm{~N} / \mathrm{A}$ \\
\hline 501 & 32.61 & $\mathrm{~N} / \mathrm{A}$ \\
\hline 502 & 33.18 & 33.26 \\
\hline
\end{tabular}

501th packet. It means that the receiver's buffer has already received the first 500 packets but not finished processing them. And at the same time, it receives the 501th packet. Since interpolation rate is not the same as the first 500 packets, the receiver cannot decode the 501th packet. Because of rate changing, receiver stops processing and empties the buffer that stores unprocessed packets (i.e. 495-500). After that, the receiver received the 502th packet and decoded it as normal.

\subsubsection{Lengthen Coordinator Scheme Design}

With the two main delays we discussed above, how to reduce the overhead is a big challenge. Lengthen Coordinator is a tricky scheme. Instead of directly avoiding these overheads, it leverage these two delays to cancel with each other.

To illustrate this, we also use table 3 as reference. After the sender emitted the 500th packet with original interpolation rate, it finished its rate changing process in around 130us. At this time, the receiver finished processing the 494th packet. Because the delay between sender and receiver is caused by both sides, it needs nearly half of the total delay time (i.e. $0.08 / 2=0.04 \mathrm{~ms}$ ) for the receiver to process one packet. Thus the receiver needs $0.04 m s \times 6=240 u s$ to finish processing the last 6 packets in its buffer. Given this, the sender prepares the 501th packet and doesn't send it, this period costs 40us. At this time the receiver has processed packet 495 . The sender waits for another $0.04 \mathrm{~ms} \times 5=200 \mathrm{us}$ which is the time for the receiver to finish processing from the 496th packet to the 500th packet. After this, the sender begins to send the 501th packet with changed rate. The receiver received this packet and began to coordinate with the sender's current interpolation rate. So there is only one packet (i.e. the 501th packet) loss rather than 7 (i.e. the 495th-500th packets with original rate and the 501th packet with changed rate) loss. Additionally, at the receiver side, there is no noticeable delay because the receiver received the following packet (i.e. the 501th packet) right after it processed the packets (i.e. the 495th-500th packet) in its buffer. In receiver's view, it is as if the transmission has no delay since it always has packets received need to be processed.

By leveraging processing delay and rate changing delay to cancel each other, we successfully reduce the overhead of implementing TiM into real-world systems. 

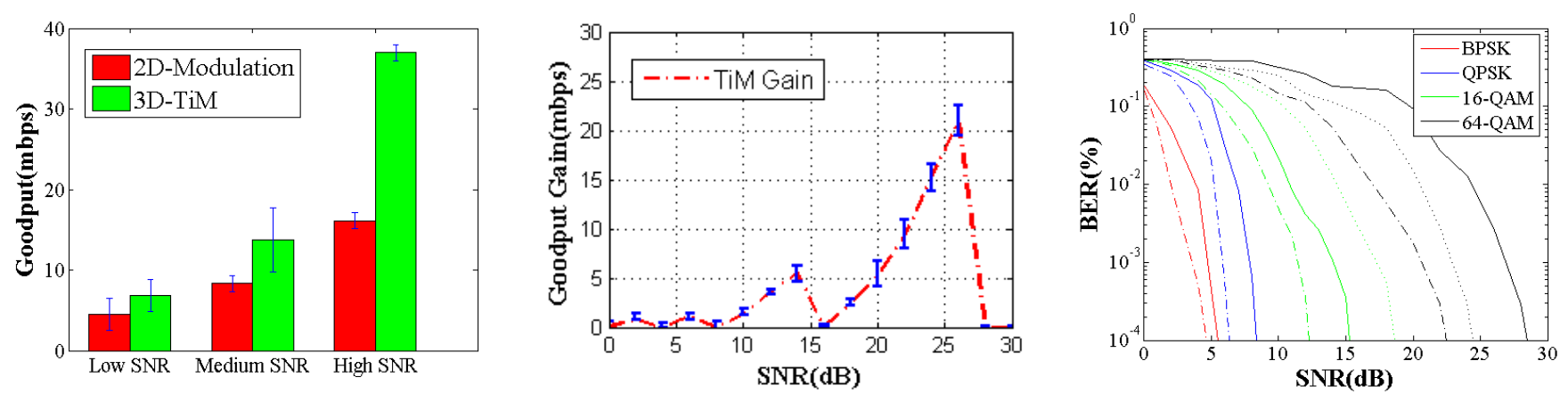

Fig. 10. Goodput comparison between traditional Fig. 11. TiM's goodput gain compared with Fig. 12. TiM's BER performance with varied S2D modulation schemes and 3D TiM with varied rate adaptation using traditional 2D modulation NR.

SNR. schemes.

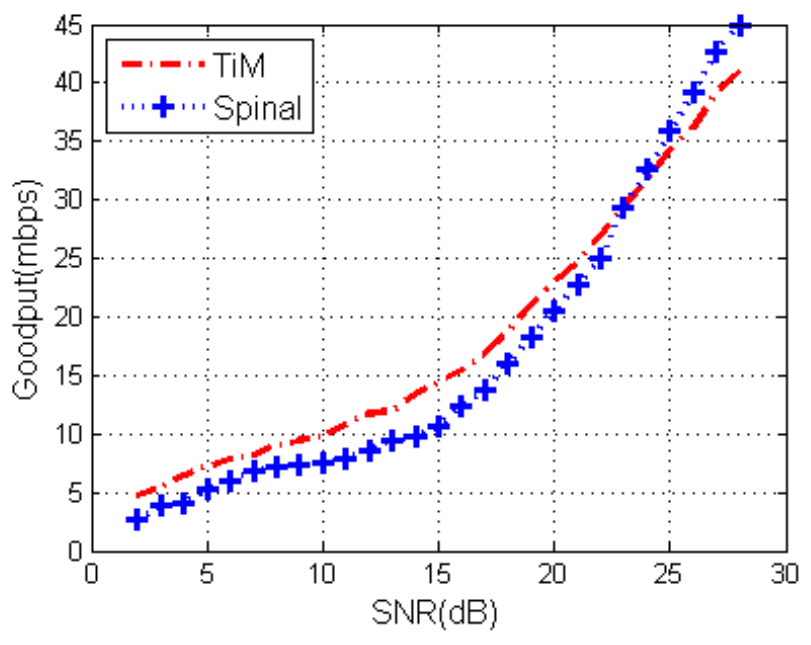

Fig. 13. Goodput comparison between TiM and rateless code (Spinal codes) in varied SNR scenarios.

\subsection{Grain Size Estimation}

Only based on the basic model of TiM, it cannot be implemented into real-world wireless networks. Before implementing TiM, we should first define how dense the finegrained level we set. Grain Size Estimation can achieve this by measuring channel variation range and frequency.

Before implementing TiM, we first make a training process of channel condition estimation. Based on the channel variation information we collect, we analysis its variation range and frequency. The basic principle is that the larger variation range and the higher variation frequency, we set less fine-grained levels (and vice versa) to meet with the trade-off between rate changing overhead and fine-grained throughput gains. Additionally, after implementing TiM, we can further adapt fine-grained level dynamically if the channel condition changes dramatically.

\section{Evaluation}

We evaluate TiM's performance in this section. Here we mainly focus on channel utilization, Lengthen Coordinator effect, fine-grain level effect, the transceivers' moving effect and energy consumption of TiM. The experimental results demonstrate that TiM can achieve up to $200 \%$ channel utilization efficiency. Furthermore, without Lengthen Coordinator the overall goodput will decrease. Additionally, Gain Size Estimation have a significant impact on the performance of TiM.

\subsection{Channel Utilization}

In this part, we evaluate TiM's performance mainly from three aspects, namely the channel utilization, bit error rate (BER) and how the nearly linear rate changing that TiM can achieve.

First, we compare the goodput (not throughput) of TiM with conventional 2D modulation in three different scenarios, namely Low SNR (ranging from 0-10 dB), Medium SNR (10-20 dB), High SNR (20-30 dB). Note that each 2D scheme (e.g. BPSK) may have different coding rates (e.g. $1 / 23 / 4)$, here we pick up the coding rate that can achieve the highest goodput as the coding rate for this specific $2 \mathrm{D}$ scheme. And we use the highest goodput to represent each scheme's performance. In addition, the performance of all the 2D schemes in Fig.10, Fig.11, Fig.14 and Fig.15 follows the same rules.

As shown in Fig.10, in low and medium SNR, TiM outperforms 2D schemes to nearly $60 \%$ on average. In high SNR, TiM can achieve up to $200 \%$ goodput compared with 2D schemes.

To make it clear, Fig.11 depicts TiM's gain over traditional 2D schemes with the SNR ranging from 0-30 dB. The gain achieves its peak value in the SNR interval of two adjacent 2D schemes. Further, the peak value increases from sparse schemes (e.g. BPSK) to dense schemes (e.g. 64-QAM). It means that the denser $2 \mathrm{D}$ modulation scheme we use, the larger gap will exist in rate adaptation. This result also exposes the defect of matching gap between 2D schemes and continuous varied channel condition. TiM can cover up this gap to improve channel utilization.

Second, we compare TiM's performance with a state-ofthe-art rateless coding scheme, namely Spinal Codes [21]. The implementation of Spinal Codes follows the configurations which are indicated in [21]. More specifically, the sender utilizes one-at-a-time type to implement hash function $h$ and corresponding random number generator RNG. For every $\mathrm{k}$ bits message $\left(\{0,1\}^{k}\right)$, we encodes the bits into one rateless symbol according to equation 21 and 22:

$$
h:\{0,1\}^{k} \times\{0,1\}^{v} \rightarrow\{0,1\}^{v}
$$




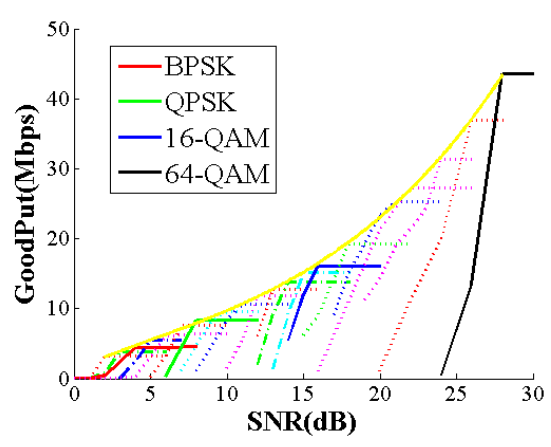

Fig. 14. TiM's nearly linear rate changing performance with varied SNR.
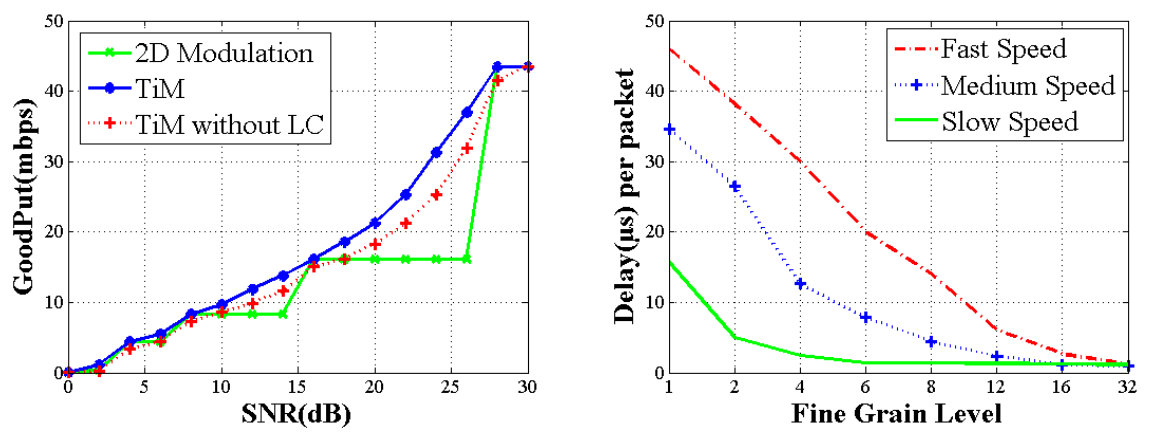

Fig. 15. Performance of TiM with/without Length- Fig. 16. TiM's fine-grained level changing delay. en Coordinator(LC) in varied SNR.

$$
R N G:\{0,1\}^{v} \times \mathbb{N} \rightarrow\{0,1\}^{c}
$$

As shown in equation $2122,\{0,1\}^{v}$ refers to a $v$-bit state of spine value. $\{0,1\}^{c}$ is the modulated rateless symbol we need.

We puncture every pass of 32 rateless symbols into 8 subpass. In each sub-pass, we transmit 4 rateless symbols. One thing needs to be noticed is that, OFDM modulation scheme can transmits multiple pass of rateless symbols at one time slot (e.g. there could be 48 rateless symbols at a time when 64-point FFT is used). Therefore, we multiplex message blocks on one OFDM symbol. Thus, on each message block, we transmit 4 rateless symbols per OFDM symbol.

On the receiver side, we perform the similar MaximumLikelihood (ML) decoding function as spinal codes [21], which is illustrated as equation 23 . $H$ refers to the corresponding channel frequency response of all sub-channels. $X_{M}$ denotes the encoded symbols of block $M$, whereas $Y$ refers to the corresponding received symbols. Since the ML algorithm is known with exponential complexity, given this, we leverage the bubble decoding method in spinal codes [21] to achieve the polynomial complexity.

$$
\widehat{M}=\arg \min _{M \in\{0,1\}^{n}}\left\|Y-H X_{M}\right\|^{2}
$$

As shown in Fig.13, TiM outperforms rateless codes (i.e. Spinal Codes) in low SNR (i.e. 2-10 dB) and medium SNR (i.e. 10-20 dB) scenarios. That is because Spinal codes only strengthens the codes' robustness. TiM indeed enhances not only the code, but also the signals' resistance to noise and interference. For high SNR (i.e. 20-28 dB) scenarios, Spinal Codes outperforms TiM. The main reason is that, in high SNR scenarios, rateless codes can use less time for decoding than fixed rate coding schemes.

Third, we get BER result with varied SNR in the experiment. In Fig.12, compared with the BER-SNR result of traditional 2D schemes, TiM makes it more dense. It means that TiM can not only fill-in the goodput gap of traditional schemes, but also cover up their BER gap. In addition, Fig.12 also verifies that TiM's data lengthen process (i.e. interpolation process) indeed enhances the signal's robust to noise and interference.

Last, we test with TiM's modulation process to achieve nearly linear rate changing in rate adaptation. In USPR2 [8] experimental environment, we make the highest finegrained level we can. We can see from Fig.14 that we add
5 intermediate TiM's schemes on average between each two adjacent traditional 2D modulation schemes. The results in Fig.14 also shows that, with TiM's fine-grained modulation schemes, the rate adaptation process is no longer staircaselike, which has gap when changing from one bitrate modulation scheme to adjacent ones. On the contrary, rate adaptation can achieve nearly linear bitrate adjustment with TiM's fine-grained modulation schemes. There is no noticeable goodput loss during this fine-grained modulation scheme changing process. In other words, results in Fig.14 verifies that TiM can achieve the mapping process between TiM's 3D modulation schemes and the estimated continuous SNR value without noticeable performance loss.

\subsection{Lengthen Coordinator Effect}

As depicted in Fig.15, there exists difference between the performance of TiM with/without Lengthen Coordinator (LC). In some circumstances, the goodput performance of TiM without LC really drops a lot which is lower than traditional 2D modulation schemes. Without dealing with the two main kinds delays properly will cause significant loss of TiM's overall goodput.

However, as a whole, TiM without LC can still outperform over the traditional 2D schemes because that the finegrained gain of TiM is larger than delay overhead in most of the circumstances. We can deduce that sometimes if we cannot implement LC in real-world scenarios, TiM can still achieve better performance than traditional 2D schemes in most of the circumstances.

\subsection{Grain Size Effect}

We evaluate the module Grain Size Estimation's effect on TiM's performance. We mainly focus on two aspects. First, we discuss about the time delay that different fine-grained levels will cause. The prime results show that the more finegrain level we set, the higher delay will cost. Second, we explore the nodes' movement effect on different fine-grain level schemes. We find out that the coarser grain level we use, the more robust to the moving scenarios and vice versa.

We measure the time delay of different fine-grained levels with the same quantity of packets for transmission. And we calculate the average time delay per packet. In Fig.16, the number on horizontal axis represents the finegrain level of TiM. For example, if the value on horizontal 

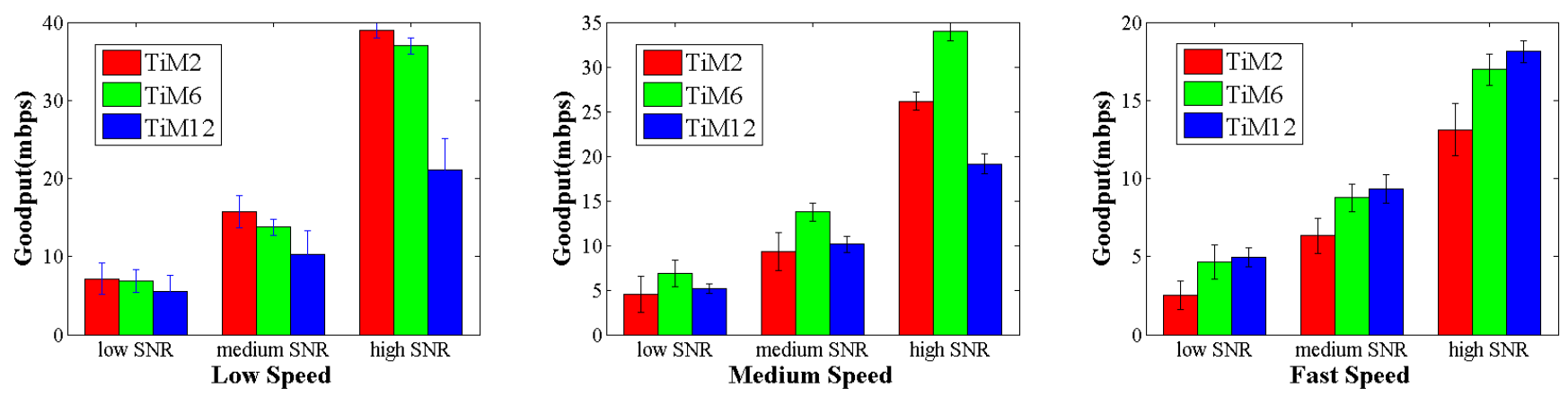

Fig. 17. Moving speed effects on TiM's performance with different fine-grained level and varied SNR.

axis of Fig.16 is 1, it means that the minimum rate changing unit is 1 (i.e. suppose the original interpolation factor is 6, with fine-grain level of 1 , the factor can be changed to 5,7 and so on). From Fig.16, we can find out that the higher finegrained level we set, the more delay overhead will cause because of the frequently rate changing.

We also test the delay with transceivers movement of different speeds. As for normal walking or little running is within 1-9 $\mathrm{mph}$ [18], we evaluate different fine-grain level with moving speed ranging from 1-9 $\mathrm{mph}$. More precisely, we divide the moving speed to three level, namley low speed (1-3 mph), medium speed (4-6 mph) and high speed (7-9 mph). The speed specification of Fig.17 follows the same rule. As shown in Fig.16, with the assistance of LC, the delay overhead decreases to a relatively acceptable value when we use fine-grained level coarser than 6 in all three kinds of moving speed. Given this, we can deduce that typically the minimum changing unit of the interpolation factor can be set to 6 with acceptable overhead when facing with varied moving speeds.

Fig.17 illustrates the varied moving impact on TiM from the goodput perspective. It depicts detailed experimental results of TiM's goodput performance with different finegrained level TiM in varied SNR and different moving speed. As shown in Fig.17, even though the high finegrained (i.e. TiM2 with minimum rate changing unit of 2) scheme has higher performance than others when the transceivers are relatively stable (i.e. low moving speed and high SNR), the overall performance of the medium finegrained scheme (i.e. TiM6 with minimum rate changing unit of 6) is the best. This result also verified that we need to make a trade-off between the gain of the TiM's fine-grained level and the overhead caused by frequently rate changing.

\subsection{Energy Consumption}

In this part, we evaluate the energy consumption of TiM compared with traditional 2D modulation schemes for transmitting the same amount of data. The sender continuously transmits a fixed $10 \mathrm{MB}$ file to the receivers. We measure the energy consumption of USRP2 sender and receiver directly via oscilloscope and current voltage probes. We conduct the 10 times power measurement for different packet size scenarios. Then we average results for each packet size scenario. The experimental SNR is roughly between 10-25 dB. We compare the energy consumption between

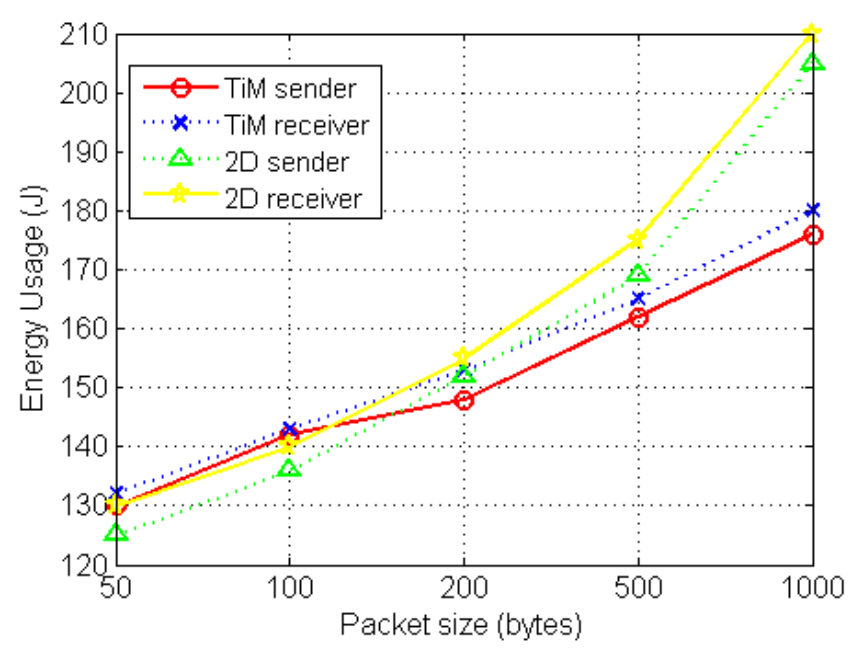

Fig. 18. Energy consumption of TiM compared with traditional 2D schemes for transmitting a $10 \mathrm{MB}$ file.

TiM's transceivers and transceivers with conventional 2D modulation.

As shown in Fig.18, the energy cost of TiM is higher than traditional 2D schemes when the packet size is relatively small (i.e. 50, 100 bytes). This is because with small packet, TiM needs more processing power for interpolation and decimation. In commonly used large packets scenarios (i.e. $200,500,1000$ bytes), the energy cost of TiM is much lower than that of traditional 2D schemes. The main reason is that, compared with traditional 2D schemes, TiM can achieve higher channel utilization and less retransmission. Thus it can complete the file delivery in less time.

\subsection{Summary of Results}

Our comprehensive experiments and simulations reveals the following key insights:

- The goodput of TiM outperforms traditional 2D schemes to nearly $60 \%$ on average. TiM can achieve up to $200 \%$ goodput compared with 2D schemes in high SNR scenarios (i.e. 20-30 dB).

- Since TiM's base-band data lengthen process can enhance signal's robustness, TiM can cover up the BER gap of traditional 2D schemes. 
- Incorporating Lengthen Coordinator can reduce rate changing delay, thus enhances TiM's performance.

- TiM can achieve nearly linear rate adaptations instead of traditional staircase-like ones.

- Based on our experimental results on URSP2, we recommend the minimum changing unit of the interpolation factor to be set to 6 when facing with moving speed of 1-9 $\mathrm{mph}$. The users can further adapt the minimum rate changing unit to enhance performance for their specific scenarios.

- According to our measurements, TiM achieves lower energy cost than traditional 2D schemes when the packet size is larger than 200 bytes. Thus TiM is more suitable for real world communication that the packets for transmission is usually larger than 1000 bytes. Moreover, TiM maintains reasonable energy cost even when the packet size is small.

\section{Related Work}

TiM is closely related to two kinds of literature. The first kind is rate adaptation based on 2D modulation schemes. The second category is to improve channel utilization by adapting signal width or using time interval to convey data.

\subsection{Rate Adaptation Based on 2D Modulation Schemes}

Rate adaptation in wireless networks has been a hot research topic for long time. There are a lot of papers addressing rate adaptation problems. Most of recent works can be classified into loss-based schemes and SNR-triggered ones.

Many papers estimate channel condition based on historical loss information [4] [5]. softrate [11] uses BER of received packets to estimate bitrate that channel can support. AccuRate in [22] leverages channel distortion to estimate the best rate. The authors propose RRAA in [23] that leverages loss information in short frame window to estimate channel condition. COLLIE in [4] allows the receiver to send back error packets to the sender in order to diagonalize the cause of the error.

SNR-based protocol is another estimation method for rate adaptation. Many current works focus on evaluating channel SNR to estimate bitrate [1] [2] [3] [24]. In [24], it demonstrates to use different rate adaptive modulation schemes with varied frequency band. In [25], Camp et al. propose a framework to evaluate rate adaptation schemes. And its conclusion is that trained SNR-based protocols outperform loss-based ones.

However, all of them are based on existed 2D modulation schemes. Thus the matching gap between modulation types and varied channel conditions still causes throughput loss. Only changing the modulation schemes into TiM's 3D domain will fix with this throughput loss.

Our work is also related to coding and modulation schemes. Current 802.11 [9] employs fixed rate codes such as LDPC codes [26], convolutional codes [27]. This kind of approach tries to combat temporal channel variation. Recently, rateless codes such as Raptor codes [28], Spinal codes [21] have attracted a lot of research interests. The basic idea is to let the sender generate limitless rateless symbols from a fixed number of data. Thus the receiver can continue collecting symbols until all the packets are successfully decoded. However, all these coding schemes only enhance the codes' robust to noise, whereas TiM can indeed improve the radio signals' resistance to noise and interference.

\subsection{Channel Utilization}

Channel Width Adaptation Some recent papers focus on how to utilize channel more efficiently [29] [30]. FICA in [30] proposes to achieve subchannel random access which can improve the overall channel utilization. However, it needs to use RTS/CTS (Request to Send/Clear to Send) signaling which are often disabled in commercial WLANs. On the other hand, In [29], the authors claim to use varied channel width for different bitrate transmission. However, it may narrow down width of channel bands. Thus, there will be more guard bands which decrease the channel utilization. On the contrary, TiM indeed will not cause signal width changing. Furthermore, the signal's robust to noise and interference does not improve as what TiM can achieve.

Time Interval Modulation There is another kind of approaches that instead of avoiding interference, they leverage interference to convey control information [7] [6]. In their proposals, they leverage the time interval between any two adjacent transmitted symbols to convey information. However, this kind of time domain usage does not improve signal's robust to interference and noise whereas TiM does.

\section{CONCLUSION}

In this paper, we propose a novel 3D modulation scheme TiM with additional time domain. It adds time-domain into existed modulation scheme's 2D (amplitude and phase) domain in order to fill-in the matching gap between limited modulation types and continuous varied channel conditions. Our measurement shows that TiM can achieve nearly linear rate changing during rate adaptation instead of current scheme's staircase-like performance. This makes TiM improve channel utilization up to $200 \%$ and increase signal's robust to noise and interference. Furthermore, assistance of Lengthen Coordinator and Grain Size Estimation enhances TiM's performance. Since TiM follows traditional modulation schemes, we believe TiM can be beneficial to widely deployed commercial WLANs.

\section{ACKNOWLEDGMENTS}

This research is supported in part by Program for New Century Excellent Talents in University (NCET-13-0908), Hong Kong RGC Grant HKUST16207714, Guangdong Natural Science Funds for Distinguished Young Scholar (No. S20120011468), the Shenzhen Science and Technology Foundation (Grant No. JCYJ20140509172719309), China NSFC Grant 61472259.

\section{REFERENCES}

[1] J. Zhang, K. Tan, J. Zhao, H. Wu, and Y. Zhang, "A practical snrguided rate adaptation," in Proceedings of IEEE INFOCOM, 2008.

[2] G. Holland, N. Vaidya, and P. Bahl, "A rate-adaptive mac protocol for multi-hop wireless networks," in Proceedings of ACM MobiCom, 2001. 
[3] B. Sadeghi, V. Kanodia, A. Sabharwal, and E. Knightly, "Opportunistic media access for multirate ad hoc networks," in Proceedings of ACM MobiCom, 2002.

[4] S. Rayanchu, A. Mishra, D. Agrawal, S. Saha, and S. Banerjee, "Diagnosing wireless packet losses in 802.11:separating collision from weak signal," in Proceedings of IEEE INFOCOM, 2008.

[5] J. Kim, S. Kim, S. Choi, and D. Qiao, "Cara:collision-aware rate adaptation for ieee 802.11 wlans," in Proceedings of IEEE INFOCOM, 2006.

[6] K. Wu, H. Tan, Y. Liu, J. Zhang, Q. Zhang, and L. M.Ni, "Side channel: Bits over interference," in Proceedings of ACM MobiCom, 2010.

[7] A. Cidon, K. Nagaraj, S. Katti, and P. Viswanath, "Flashback: Decoupled lightweight wireless control," in Proceedings of ACM SIGCOMM, 2012.

[8] E. R. LLC, Universal Software Radio Peripheral, http://www.ettus. com.

[9] E. Blossom, Wireless LAN Medium Access Control (MAC) and Physical Layer (PHY) Specifications, IEEE Std 802.11, 2012.

[10] K. C.-J. Lin, N. Kushman, and D. Katabi, "Ziptx: Harnessing partial packets in 802.11 networks," in Proceedings of ACM MobiCom, 2008.

[11] M. Vutukuru, H. Balakrishnan, and K. Jamieson, "Cross-layer wireless bit rate adaptation," in Proceedings of ACM SIGCOMM, 2009.

[12] F. Lu, G. M. Voelker, and A. C. Snoeren, "Slomo: Downclocking wifi communication," in Proceedings of NSDI, 2013.

[13] X. Zhang and K. G. Shin, "E-mili: Energy-minimizing idle listening in wireless networks," in Proceedings of ACM MobiCom, 2011.

[14] M. E. Frerking, Digital Signal Processing in Communication Systems. Norwell, MA, USA: Kluwer Academic Publishers, 1993.

[15] R. G. Lyons, Understanding Digital Signal Processing (2nd Edition). Upper Saddle River, NJ, USA: Prentice Hall PTR, 2004.

[16] J. Y. Kwentus, Z. Jiang, and A. N. Willson, "Application of filter sharpening to cascaded integrator-comb decimation filters," IEEE Transactions on Signal Processing, vol. 45, no. 2, 1997.

[17] E. Blossom, Gnu software defined radio, http://www.gnu.org/ software/gnuradio.

[18] S. T. Aditya and S. Katti, "Flexcast: Graceful wireless video streaming," in Proceedings of ACM MobiCom, 2011.

[19] K. Jamieson and H. Balakrishnan, "PPR: Partial packet recovery for wireless networks," in Proceedings of ACM SIGCOMM, 2007.

[20] J. Han and M. Kamber, Data Mining: Concepts and Techniques (2nd Edition). San Francisco, CA, USA: Morgan Kaufmann Publishers, 2006.

[21] J. Perry, P. A. lannucci, K. Fleming, HariBalakrishnan, and D. Shah, "Spinal codes," in Proceedings of ACM SIGCOMM, 2012.

[22] S. Sen, N. Santhapuri, R. R. Choudhury, and S. Nelakuditi, "Accurate: Constellation based rate estimation in wireless networks," in Proceedings of NSDI, 2010.

[23] S. H. Wong, H. Yang, S. Lu, and V. Bharghavan, "Robust rate adaptation for 802.11 wireless networks," in Proceedings of ACM MobiCom, 2006

[24] H. Rahul, F. Edalat, D. Katabi, and C. Sodini, "Frequency-aware rate adaptation and mac protocols," in Proceedings of ACM MobiCom, 2009.

[25] J. Camp and E. Knightly, "Modulation rate adaptation in urban and vehicular environments: Cross-layer implementation and experimental evaluation," in Proceedings of ACM MobiCom, 2008.

[26] R. Gallager, "Low-density parity-check codes," IEEE Transactions on Information Theory, vol. 8, no. 1, 1962.

[27] A. Viterbi, "Error bounds for convolutional codes and an asymptotically optimum decoding algorithm," IEEE Transactions on Information Theory, vol. 13, no. 2, 1967.

[28] A. Shokrollahi, "Raptor codes," IEEE Transactions on Information Theory, vol. 52, no. 6, 2006.

[29] R. Chandra, R. Mahajan, T. Moscibroda, R. Raghhavendra, and P. Bahl, "A case for adapting channel width in wireless networks," in Proceedings of ACM SIGCOMM, 2008.

[30] K. Tan, J. Fang, Y. Zhang, S. Chen, L. Shi, J. Zhang, and Y. Zhang, "Fine-grained channel access in wireless lan," in Proceedings of ACM SIGCOMM, 2010.

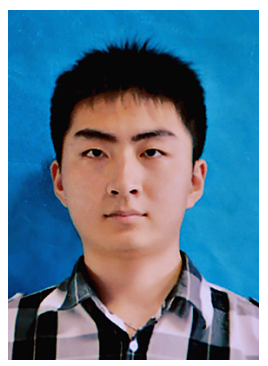

Guanhua Wang received his B.Eng. degree from Southeast University, Nanjing, China, in 2012 , and is currently a master of philosophy student in computer science and engineering at Hong Kong University of Science and Technology. He will start pursuing his Ph.D. degree at AMPLab in Computer Science Division at UC Berkeley in Fall 2015. His main research interests include Wi-Fi radar, wireless communications and mobile computing.

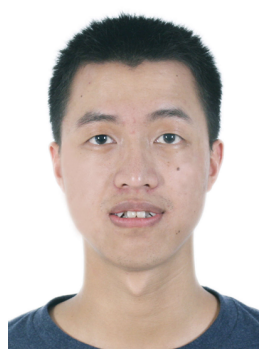

Shanfeng Zhang received his B.Eng. degree from Shanghai Jiao Tong University, Shanghai, China, in 2012, and is currently working towards the Ph.D. degree in computer science and engineering in the Hong Kong University of Science and Technology. His main research interests include wireless communications, mobile sensing and vehicular networks.

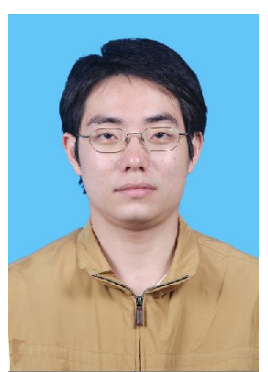

Kaishun Wu is currently a research assistant professor in Fok Ying Tung Graduate School with the Hong Kong University of Science and Technology (HKUST). He received the Ph.D. degree in computer science and engineering from HKUST in 2011. He received the Hong Kong Young Scientist Award in 2012. His research interests include wireless communication, mobile computing, wireless sensor networks and data center networks.

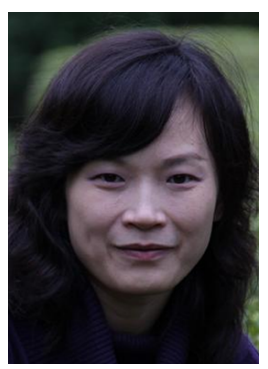

Qian Zhang received the $\mathrm{PhD}$ degree from Wuhan University, China, in 1999. She joined the Hong Kong University of Science and Technology in September 2005, where she is a full professor in the Department of Computer Science and Engineering. Her current research interests include wireless communications, IP networking, multimedia, P2P overlay, and wireless security. She is a fellow of the IEEE.

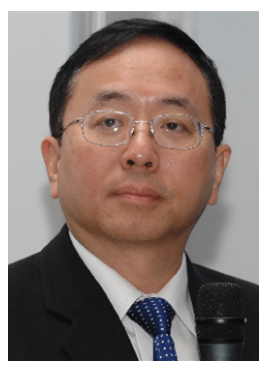

Lionel M. Ni is Chair Professor in the Department of Computer and Information Science and Vice Rector of Academic Affairs at the University of Macau. Previously, he was Chair Professor of Computer Science and Engineering at the Hong Kong University of Science and Technology. He received the Ph.D. degree in electrical and computer engineering from Purdue University in 1980. A fellow of IEEE and Hong Kong Academy of Engineering Science, Dr. Ni has chaired over 30 professional conferences and has received eight awards for authoring outstanding papers. 